\title{
Stroke Prevalence, Mortality and Disability-Adjusted Life Years in Adults Aged 20-64 Years in 1990-2013: Data from the Global Burden of Disease 2013 Study
}

\author{
Rita V. Krishnamurthi ${ }^{a}$ Andrew E. Moran ${ }^{f}$ Valery L. Feigin ${ }^{a}$ \\ Suzanne Barker-Collo ${ }^{c}$ Bo Norrving ${ }^{d}$ George A. Mensahe $^{\mathrm{e}}$ Steve Taylor $^{\mathrm{b}}$ \\ Mohsen Naghavig Mohammed H. Forouzanfarg Grant Nguyen ${ }^{\text {h }}$ \\ Catherine O. Johnson $^{\text {h }}$ Theo Vos ${ }^{g}$ Christopher J.L. Murrayg Gregory A. Roth ${ }^{\text {h, i }}$ \\ for the GBD 2013 Stroke Panel Experts Group
}

${ }^{a}$ National Institute for Stroke and Applied Neurosciences, and bepartment of Biostatistics and Epidemiology, Auckland University of Technology, and ' School of Psychology, The University of Auckland, Auckland, New Zealand; ${ }^{\mathrm{d} D e p a r t m e n t}$ of

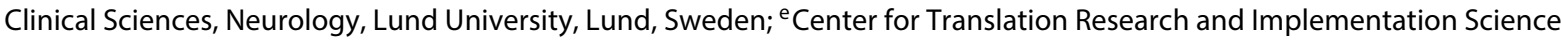
and Division of Cardiovascular Sciences; National Heart, Lung, and Blood Institute; National Institutes of Health, Bethesda, Md., f Division of General Medicine, Columbia University, New York, N.Y., ${ }^{9}$ Department of Global Health, School of Medicine and Public Health, University of Washington, ${ }^{\mathrm{h}}$ Institute for Health Metrics and Evaluation, and 'Division of Cardiology,

School of Medicine, University of Washington, Seattle, Wash., USA

\section{Key Words}

Stroke · Ischemic · Hemorrhagic · Young adult · Global trends $\cdot$ Prevalence $\cdot$ Deaths $\cdot$ DALYs

\footnotetext{
Abstract

Background: Recent evidence suggests that stroke is increasing as a cause of morbidity and mortality in younger adults, where it carries particular significance for working individuals. Accurate and up-to-date estimates of stroke burden are important for planning stroke prevention and management in younger adults. Objectives: This study aims to estimate prevalence, mortality and disability-adjusted life years (DALYs) and their trends for total, ischemic stroke (IS) and hemorrhagic stroke (HS) in the world for 1990-2013 in adults aged 20-64 years. Methodology: Stroke prevalence, mortality and DALYs were estimated using the Global Burden of Disease (GBD) 2013 methods. All available data on rates of stroke incidence, excess mortality, prevalence and
}

death were collected. Statistical models were used along with country-level covariates to estimate country-specific stroke burden. Stroke-specific disability weights were used to compute years lived with disability and DALYs. Means and 95\% uncertainty intervals (UIs) were calculated for prevalence, mortality and DALYs. The median of the percent change and $95 \% \mathrm{UI}$ were determined for the period from 1990 to 2013. Results: In 2013, in younger adults aged 20-64 years, the global prevalence of HS was 3,725,085 cases ( $95 \%$ UI $3,548,098-3,871,018)$ and IS was $7,258,216$ cases $(95 \%$ UI 6,996,272-7,569,403). Globally, between 1990 and 2013, there were significant increases in absolute numbers and prevalence rates of both $\mathrm{HS}$ and IS for younger adults. There were $1,483,707$ (95\% UI 1,340,579-1,658,929) stroke deaths globally among younger adults but the number of deaths

Members of the GBD 2013 Stroke Panel Experts Group are listed in the Appendix at the end of the paper.

\section{KARGER 125}

(c) 2015 S. Karger AG, Base

$0251-5350 / 15 / 0453-0190 \$ 39.50 / 0$

E-Mail karger@karger.com

www.karger.com/ned
Rita V. Krishnamurthi

Auckland University of Technology

90 Akoranga Drive

Northcote 0627, Auckland (New Zealand)

E-Mail rita.krishnamurthi@aut.ac.nz 
from HS $(1,047,735$ (95\% UI 945,087-1,184,192)) was significantly higher than the number of deaths from IS $(435,972$ $(95 \%$ UI 354,018-504,656)). There was a $20.1 \%$ (95\% UI -23.6 to -10.3 ) decline in the number of total stroke deaths among younger adults in developed countries but a $36.7 \%$ (95\% UI 26.3-48.5) increase in developing countries. Death rates for all strokes among younger adults declined significantly in developing countries from 47 (95\% UI 42.6-51.7) in 1990 to 39 (95\% UI 35.0-43.8) in 2013. Death rates for all strokes among younger adults also declined significantly in developed countries from 33.3 (95\% UI 29.8-37.0) in 1990 to 23.5 (95\% UI 21.1-26.9) in 2013. A significant decrease in HS death rates for younger adults was seen only in developed countries between 1990 and 2013 (19.8 (95\% UI 16.9-22.6) and 13.7 (95\% UI 12.1-15.9)) per 100,000). No significant change was detected in IS death rates among younger adults. The total DALYs from all strokes in those aged 20-64 years was $51,429,440$ (95\% UI 46,561,382-57,320,085). Globally, there was a $24.4 \%$ (95\% UI 16.6-33.8) increase in total DALY numbers for this age group, with a $20 \%$ (95\% UI 11.7-31.1) and $37.3 \%$ (95\% UI 23.4-52.2) increase in HS and IS numbers, respectively. Conclusions: Between 1990 and 2013, there were significant increases in prevalent cases, total deaths and DALYs due to HS and IS in younger adults aged 20-64 years. Death and DALY rates declined in both developed and developing countries but a significant increase in absolute numbers of stroke deaths among younger adults was detected in developing countries. Most of the burden of stroke was in developing countries. In 2013, the greatest burden of stroke among younger adults was due to HS. While the trends in declining death and DALY rates in developing countries are encouraging, these regions still fall far behind those of developed regions of the world. A more aggressive approach toward primary prevention and increased access to adequate healthcare services for stroke is required to substantially narrow these disparities.

(c) 2015 S. Karger AG, Basel

\section{Introduction}

The burden of stroke in young adults aged 20-64 years has recently come to greater attention with studies showing an increase in the incidence of stroke in young adults over the last 3 decades [1-3]. While accurate comparisons are difficult between studies due to varying age ranges used for 'young stroke', approximately $5-10 \%$ of strokes are thought to occur in people under the age of 50 years [4]. According to the Global Burden of Disease (GBD) 2010 estimates, young adults aged $20-64$ years constitut-

Stroke Prevalence, Mortality and DALYs in Adults Aged 20-64 Years in 1990-2013 ed $31 \%$ of incident strokes globally [5]. A systematic review of literature on young stroke (between 20 and 44 years) suggests that stroke in those younger than 45 years is not as uncommon as previously perceived with standardized incidence rates ranging from 8.7 to 21.0 per 100,000 [6]. Evidence suggests that changes in unfavorable lifestyle factors such as unhealthy diets high in sugar, salt and processed foods, smoking, alcohol intake, drug use and reduced levels of physical activity have led to the increased exposure to stroke risk factors in the young [7, 8]. A population-based study in the United States found an increase in the number of young people aged 18-54 years with stroke, and that over half of these were current smokers and 1 in 5 abused illegal drugs [9]. The long-term impact of stroke is large in younger adults due to the impact of lost healthy life years among working-age adults, given their contributions and responsibilities as wageearners and care-givers. Therefore, estimating the extent of the burden and temporal trends in younger adults with stroke is of critical importance for measuring societal impact and for data-driven public health planning and resource allocation.

\section{Methods}

Stroke modeling and methods used to estimate all-cause mortality, stroke-specific mortality, disability and prevalence have been previously described $[10,11]$. In brief, all available data on mortality were corrected for non-specific codes and used to estimate mortality rates for GBD causes of death, including ischemic stroke (IS) and hemorrhagic stroke (HS). An ensemble modeling method was used to estimate death rates by age, sex, year and country [12]. Disease prevalence was estimated using the DisMod-MR disease modeling software [13]. We used all available estimates of stroke incidence, prevalence and case fatality from systematic reviews of the scientific literature, population surveys and stroke registries as well as GBD mortality estimates. Disability due to acute stroke was considered to last for 28-30 days while chronic stroke lasted from 30 days until death. Disability-adjusted life years (DALYs) were calculated as the sum of years of life lost prematurely due to stroke and years lived with disability due to stroke. Countries were defined as developing or developed according to GBD methods.

\section{Results}

\section{Prevalence}

$G B D$ 2013. Among adults age 20-64 years, the global prevalence of HS in 2013 was 3,725,085 (95\% uncertainty interval (UI) 3,548,098-3,871,018) and prevalence of IS was 7,258,216 (95\% UI 6,996,272-7,569,403; table 1). The 
Table 1. Prevalent cases, deaths and DALYs in those aged 20-64 years between 1990 and 2013

\begin{tabular}{lcclccrr}
\hline & Year & Prevalence & 95\% UI & Death & \multicolumn{2}{l}{$95 \%$ UI } & \multicolumn{2}{l}{ DALYs } & $95 \%$ UI \\
\hline HS & & & & & & \\
Developing & 1990 & $1,450,240$ & $1,391,277-1,510,464$ & 716,996 & $636,079-797,977$ & $25,688,495$ & $22,803,083-28,597,621$ \\
& 2013 & $2,665,770$ & $2,521,485-2,789,120$ & 935,939 & $839,444-1,060,482$ & $32,834,364$ & $29,352,075-37,133,284$ \\
\hline Developed & 1990 & 530,589 & $490,269-574,953$ & 140,931 & $119,814-160,849$ & $4,839,572$ & $4,165,098-5,492,067$ \\
& 2013 & $1,059,315$ & $983,851-1,138,970$ & 111,795 & $98,625-129,473$ & $3,861,930$ & $3,426,825-4,432,344$ \\
\hline Global & 1990 & $1,980,830$ & $1,918,964-2,056,065$ & 857,927 & $761,615-955,263$ & $30,528,067$ & $27,119,019-33,944,576$ \\
& 2013 & $3,725,085$ & $3,548,098-3,871,018$ & $1,047,735$ & $945,087-1,184,192$ & $36,696,295$ & $33,011,678-41,372,106$ \\
\hline IS & & & & & & \\
Developing & 1990 & $1,724,774$ & $1,632,729-1,810,733$ & 225,925 & $181,026-266,063$ & $7,573,447$ & $6,058,331-8,960,467$ \\
& 2013 & $3,214,108$ & $3,033,261-3,446,309$ & 356,408 & $280,551-414,875$ & $11,769,652$ & $9,345,527-13,601,182$ \\
\hline Developed & 1990 & $2,076,621$ & $1,954,109-2,206,981$ & 95,635 & $78,800-112,305$ & $3,157,115$ & $2,664,748-3,664,830$ \\
& 2013 & $4,044,107$ & $3,869,281-4,238,562$ & 79,564 & $68,651-96,978$ & $2,963,492$ & $2,551,752-3,520,588$ \\
\hline Global & 1990 & $3,801,396$ & $3,660,560-3,957,700$ & 321,560 & $259,801-370,056$ & $10,730,563$ & $8,685,007-12,392,179$ \\
& 2013 & $7,258,216$ & $6,996,272-7,569,403$ & 435,972 & $354,018-504,656$ & $14,733,144$ & $12,209,576-17,011,339$ \\
\hline
\end{tabular}

global prevalence rates were 90.3 (95\% UI 86.0-93.8) and 176 (95\% UI 169.6-183.5) per 100,000 for HS and IS, respectively.

Developing vs. Developed Countries. In 2013, the prevalence rate of HS was significantly higher in developed countries (130.1 (95\% UI 120.8-139.9)) than in developing countries (80.5 (95\% UI 76.2-84.2)). The prevalence rate of IS was also significantly higher (by about 5-fold) in developed countries (496.7 (95\% UI 475.3-520.6)) compared to that in developing countries (97.1 (95\% UI 91.6-104.1)). In 2013, the absolute number of prevalent HS cases in developed countries $(1,059,315$ (95\% UI 983,851-1,138,970)) was 5-fold higher than that of IS $(2,665,770$ (95\% UI $2,521,485-2,789,120))$, with greatest differences seen in those aged 50-59 years. In contrast, the number of prevalent HS cases was similar or lower than prevalent IS across age groups in developing countries (table 1).

By GBD Regions. The highest prevalence rates of both IS and HS were seen in seen high-income regions of Asia Pacific, North America, East and Southeast Asia, in those aged 50-64 years. The lowest prevalence of HS was seen in North Africa and the Middle East, while the lowest prevalence of IS was seen Central Latin America, with lowest rates in those of age 20-29 years.

Trends 1990-2013. Globally, there were significant increases in both numbers of cases (table 1) and prevalence rates of both HS and IS between 1990 and 2013 (fig. 1). The number of prevalent HS cases increased significantly in developing countries between $1990(1,450,240$ (95\% UI $1,391,277-1,510,464))$ and $2013(92,665,770$ (95\% UI $2,521,485-2,789,120))$. Similarly, the absolute number of prevalent IS cases also increased significantly in developing countries between 1990 (1,724,774 (95\% UI 1,632,729$1,810,733))$ and $2013(3,214,108$ (95\% UI 3,033,261$3,446,309)$ ). In developed countries, the number of prevalent HS cases increased from 530,589 (95\% UI $490,269-574,953)$ in 1990 to $1,059,315$ (95\% UI 983,851$1,138,970)$ in 2013 while the number of prevalent IS cases increased significantly from 2,076,621 (95\% UI 1,954,109$2,206,981$ ) in 1990 to $4,044,107$ (95\% UI $3,869,281-$ $4,238,562)$ in 2013.

The global prevalence of HS increased almost 2-fold from $1,980,830$ (95\% UI $1,918,964-2,056,065)$ cases in 1990 to 3,725,085 (95\% UI 3,548,098-3,871,018) in 2013. There was a similar nearly 2 -fold increase in IS from $3,801,396$ (95\% UI 3,660,560-3,957,700) cases in 1990 to $7,258,216$ (95\% UI 6,996,272-7,569,403) in 2013. In developed countries, the prevalence rate of HS increased significantly by 1.7 -fold between 1990 (74.6 (95\% UI 69.0-80.9)) and 2013 (130.1 (95\% UI 120.8-139.9)). In developing countries, there was a smaller (1.1-fold) but significant increase in the prevalence rate of HS between 1990 (72.3 (95\% UI 69.4-75.3)) and 2013 (80.5 (95\% UI 76.2-84.2; fig. 1). The prevalence rate of IS also increased significantly in developed countries between 1990 (292.1 per 100,000 (95\% UI 274.8-310.4)) and 2013 (496.7 per 100,000 (95\% UI 475.3-520.6)), while in developing 
Fig. 1. Trends in prevalence rates for HS and IS between 1990 and 2013.

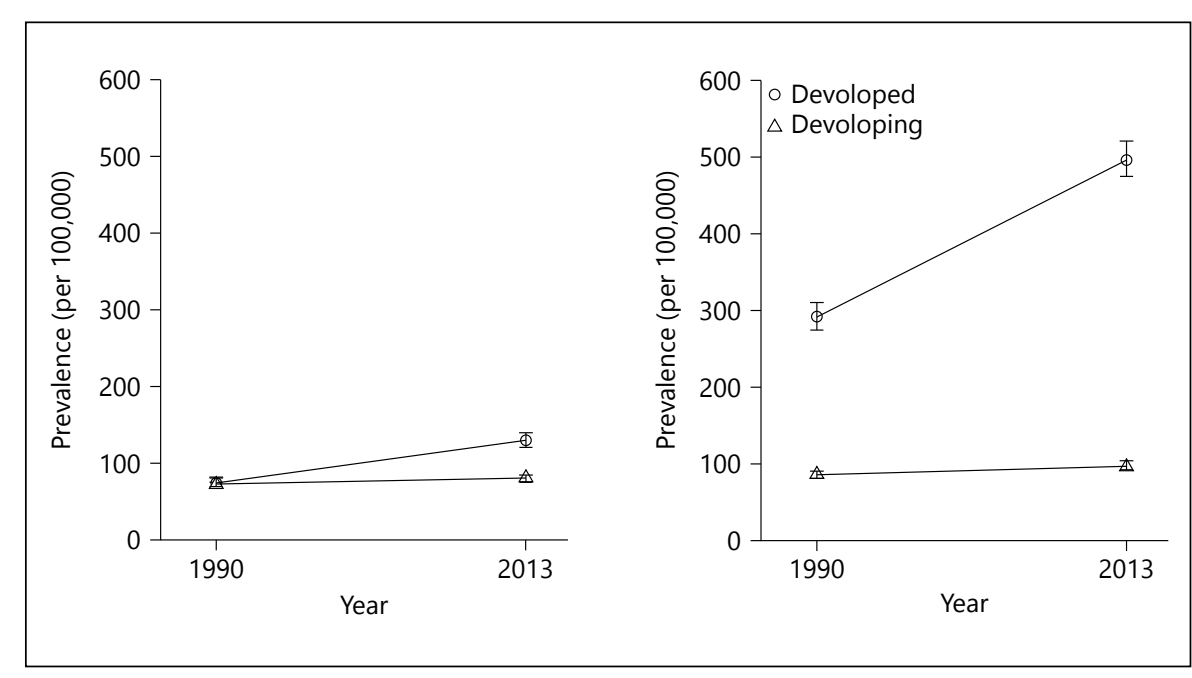

countries, the prevalence rate increased significantly from 86 (95\% UI 81.4-90.3) in 1990 to 97.1 per 100,000 (95\% UI 91.6-104.1) per 100,000 in 2013.

\section{Deaths}

GBD 2013. In 2013, there were $1,483,707$ (95\% UI $1,340,579-1,658,929)$ deaths due to stroke globally in those of age 20-64 years. The number of deaths from HS $(1,047,735$ (95\% UI 945,087-1,184,192)) was significantly higher than the number of deaths from IS $(435,972$ (95\% UI 354,018-504,656; table 1)).

Developing vs. Developed and by Region. The number of deaths as well the death rate was significantly higher in developing countries compared to developed countries (death rates 39 (95\% UI 35.0-43.8) and 23.5 (95\% UI 21.1-26.9) per 100,000) for developing and developed countries, respectively. The lowest death rates were in Australasia (Australia and New Zealand), and highest death rates were seen in South and East Asia, both for IS and HS, with the oldest individuals carrying the highest risk of stroke-related death.

Trends 1990-2013. Globally, there was a $25.6 \%$ (95\% UI 17.7-35.2) increase in the number of prevalent stroke cases, with a 21.8 (95\% UI 13.1-34.1) and 35.7\% increase in HS and IS cases, respectively. However, there was a $20.1 \%$ (95\% UI -23.6 to -10.3 ) decline in total stroke deaths in developed countries, particularly in the younger age groups (20-44 years) but a $36.7 \%$ (95\% UI $26.3-$ 48.5) increase in developing countries (fig. 2 and online suppl. appendix table 1; for all online suppl. material, see www.karger.com/doi/10.1159/000441098). Death rates for all strokes declined significantly in developed countries from 33.3 (95\% UI 29.8-37.0) in 1990 to 23.5 (95\%
UI 21.1-26.9) per 100,000 in 2013. Death rates for all strokes showed a non-significant trend to decline in developing countries from 47 (95\% UI 42.6-51.7) in 1990 to 39 (95\% UI 35.0-43.8) per 100,000 in 2013 (fig. 3). A significant decrease in death rates was seen in HS and only in between 1990 (19.8 (95\% UI 16.9-22.6)) and 2013 (13.7 (95\% UI 12.1-15.9)) per 100,000. Increases in deaths in developing countries were seen particularly in those aged 45-64 years (fig. 3). Significant declines on mortality were seen in the high-income regions of Asia Pacific and other developed regions of the word, whereas increases in mortality were seen in African regions and Oceania across all age groups (fig. 4 ).

DALYs

GBD 2013. In 2013, total DALYs from all strokes in those aged 20-64 years was 51,429,440 (95\% UI $46,561,382-57,320,085)$. The number of DALYs from HS $(36,696,295$ (95\% UI 33,011,678-41,372,106)) was significantly higher than the number of DALYs from IS $(14,733,144$ (95\% UI 12,209,576-17,011,339)).

Developing vs. Developed and by Region. Total DALYs were significantly higher by 6.5 -fold in developing countries $(44,604,017$ (95\% UI 40,099,464-49,788,189)) compared to developed countries $(6,825,422 \quad(95 \%$ UI $6,161,482-7,716,733$; table 1). DALY rates were also significantly higher in developing countries compared to developing countries. In developing countries, total DALYs and DALY rates were significantly higher for HS (991.8 (95\% UI 886.6-1,121.6)) compared to IS (355.5 (95\% UI 282.3-410.8)), while no significant difference was seen between HS and IS rates in developed countries. The lowest DALY rates were in Australasia and highest in East 
Fig. 2. Trends in death (a) and DALY (b) rates between 1990 and 2013.

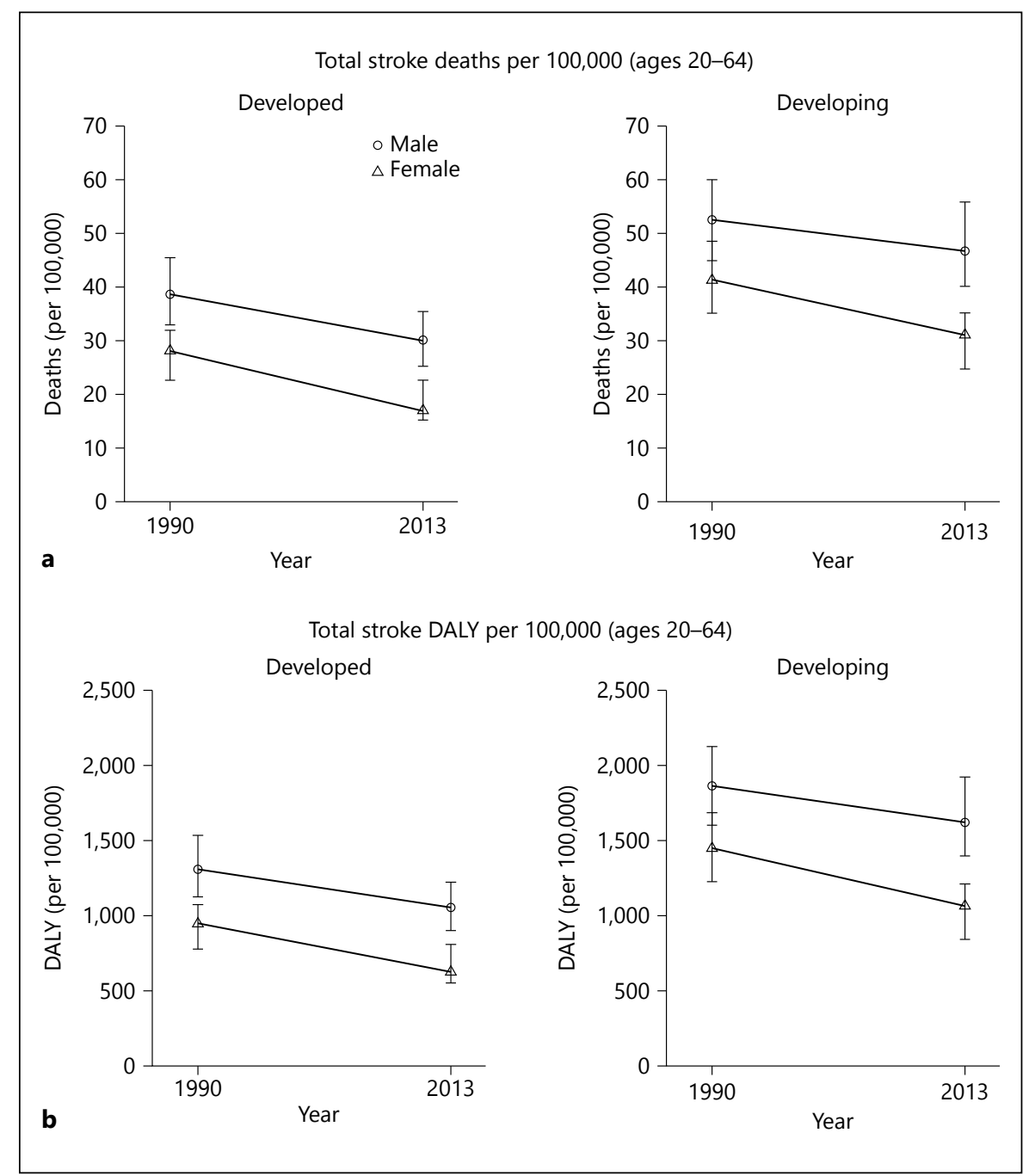

Asia, both for IS and HS. The highest rates for both stroke types were in the age group of 60-64 years (fig. 4).

Trends 1990-2013. Globally, there was a $24.4 \%$ (95\% UI $16.6-33.8$ increase in total DALY numbers, with a $20 \%$ (95\% UI 11.7-31.1) and 37.3\% (95\% UI 23.4-52.2) increase in HS and IS numbers, respectively. In developed countries, there was a $15.3 \%(95 \% \mathrm{UI}-19.3$ to -7.1$)$ decline in total DALYs while there was a 33.9\% (95\% UI 23.8-45.4) increase in developing countries. DALY rates declined significantly in developed countries from 1,124.7 (95\% UI $1,013.1-1,242.6)$ in 1990 to 838.4 (95\% UI 756.8-947.9) in 2013 (fig. 2 and online suppl. appendix table 1). DALY rates also declined in developing countries but this change was not significant. HS and IS DALY rates decreased in developing countries by $27.6 \%$ (95\% UI $17.6-40.8$ ) and $56.2 \%$ (95\% UI 33.5-77), respectively, while HS and IS DALY rates decreased by $20.6 \%$ (95\% UI -26.5 to -10.6 ) and $7 \%$ (95\% UI -17.8 to 10.0 ), respectively, in developed countries. Increases in DALYs were seen particularly in those aged 45-64 years in developing countries (fig. 3).

\section{Discussion}

The risk of strokes in adults aged 20-64 years is lower than that in older adults, but the societal impact is high due to the greater number of years of life lost and the resulting loss in productivity [14]. This study provides global estimates of stroke burden in terms of prevalence, deaths and DALYs in those aged 20-64 years by stroke type. In 2013, the burden of stroke in adults aged 20-64 years was high, with almost 11 million living with stroke and almost 1.5 million deaths from stroke, contributing to over 51 million DALYs. This represents almost half of 
Fig. 3. Percentage change in death (a) and DALYS (b) between 1990 and 2013 by age group.

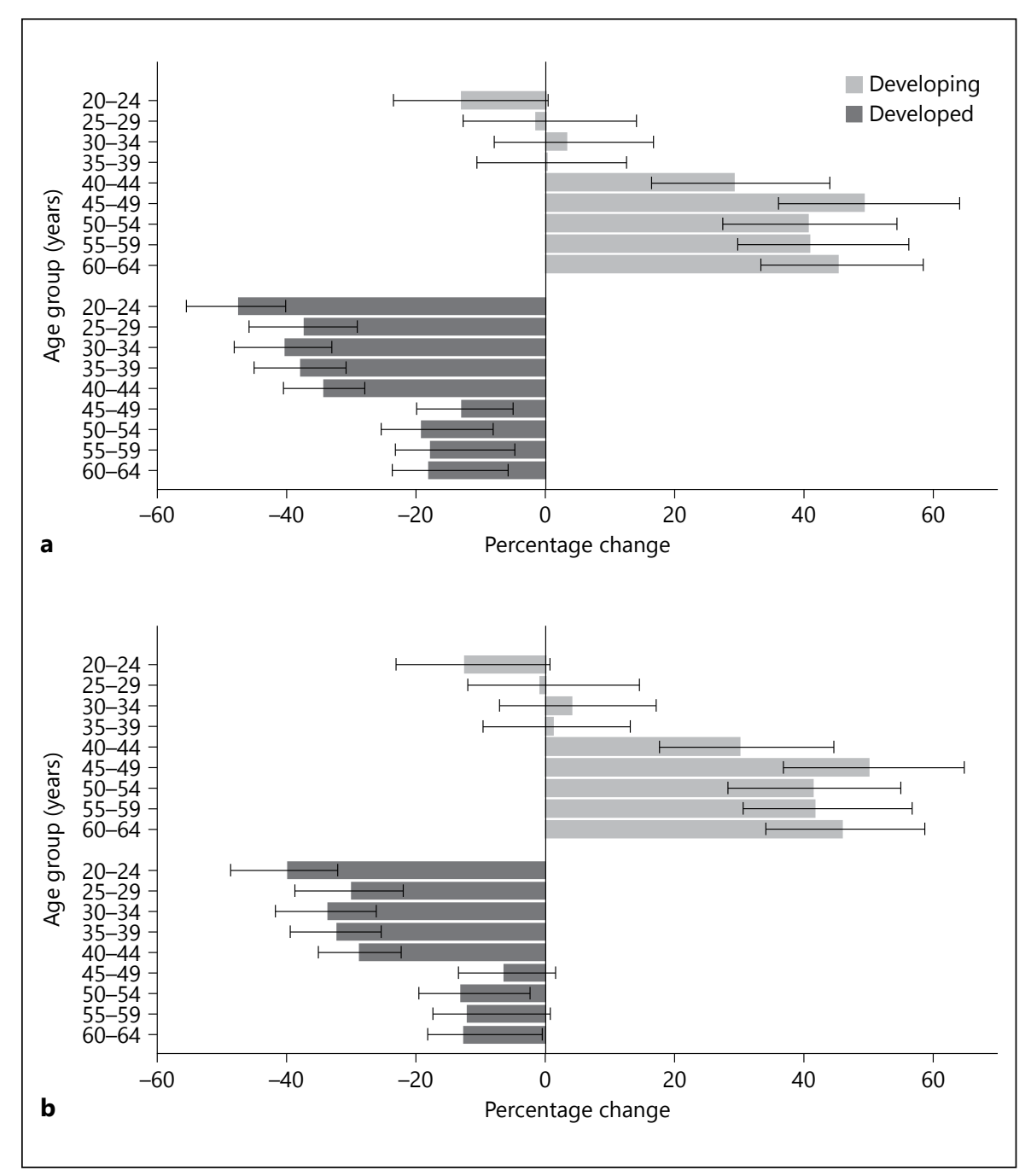

the total DALYS from stroke [10]. Significant increases were seen between 1990 and 2013 in the total numbers of prevalent HS and HS as well as total deaths and DALYs for this age group. Disparities were also found in trends of stroke burden between developing and developed countries, as death and DALY rates of stroke were significantly higher in developing countries than those in developed countries, and their rates declined significantly in developed countries but not in developing countries. Most of the burden of stroke was in developing countries, and due to HS in terms of the number of prevalent strokes, deaths and DALYS. The greater global burden of HS is likely due to greater burden of HS in developing countries where the burden of stroke is the largest.

There have been several reports of increasing stroke incidence in younger adults $[1,2,15]$, although the differing age ranges used to define this age group make it dif- ficult to compare studies [16]. Nonetheless, our data show that the overall burden of stroke in younger adults is increasing globally, with significant increases in absolute numbers of young people living with stroke and a disproportionate burden in developing countries. This has implications for healthcare and management resources, as well as plans for preventative measures to reduce this burden $[17,18]$.

It is also important to consider the etiological origins of stroke in young adults. The relative importance of modifiable risk factors may differ in young adults $[19,20]$ with cervical artery dissection [21] and cardioembolic [7] strokes known to be prominent causes of IS in younger adults and other less common etiologies such as patent foramen ovale, illicit drug use, migraine and oral contraceptives $[8,22]$ playing an important role in this age group. Antiphospholipid antibodies have also been 


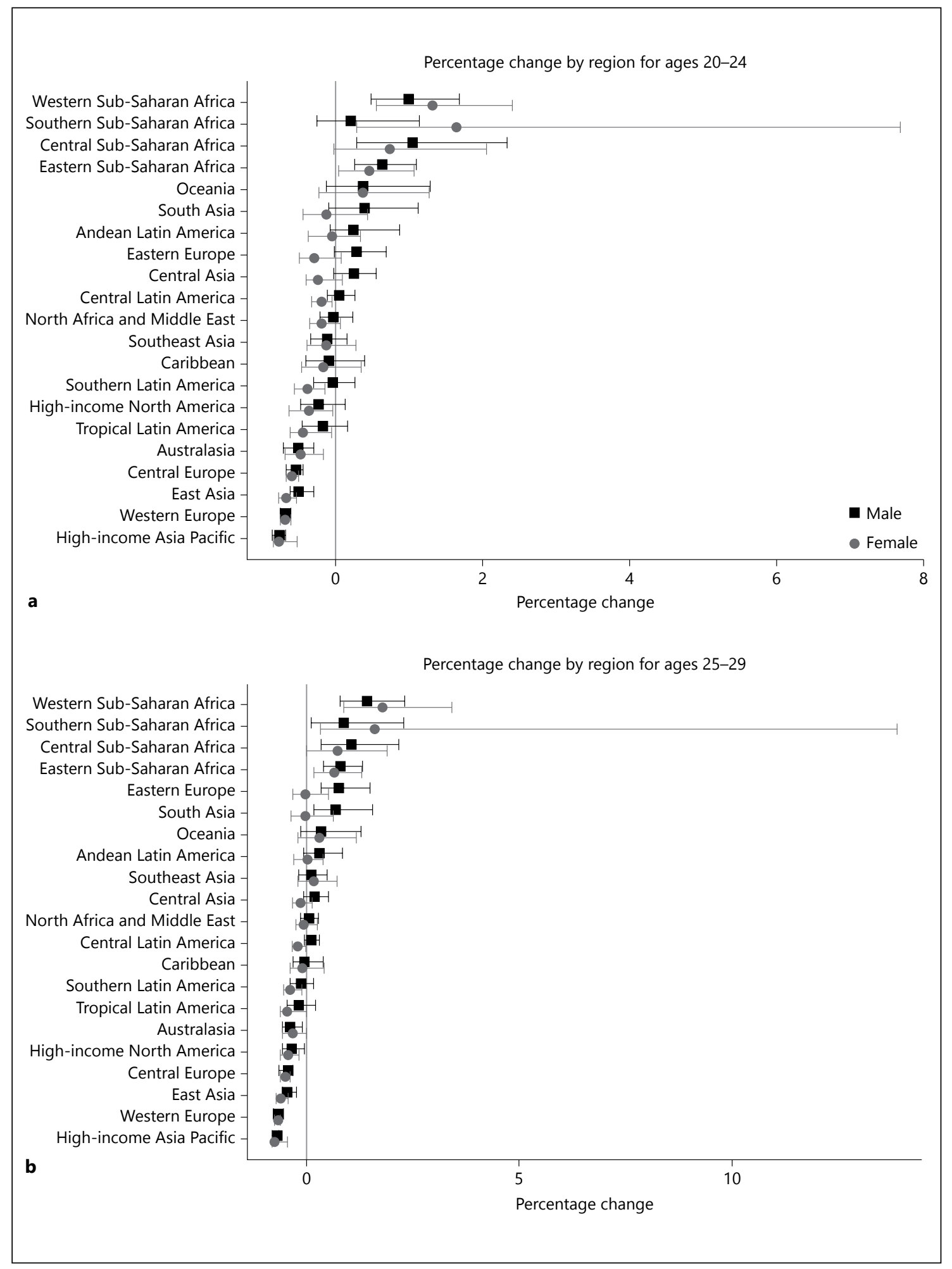

Fig. 4. a-i Percentage change in total stroke mortality by GBD region and age group.

(For figures $4 c-i$ see next pages.) 


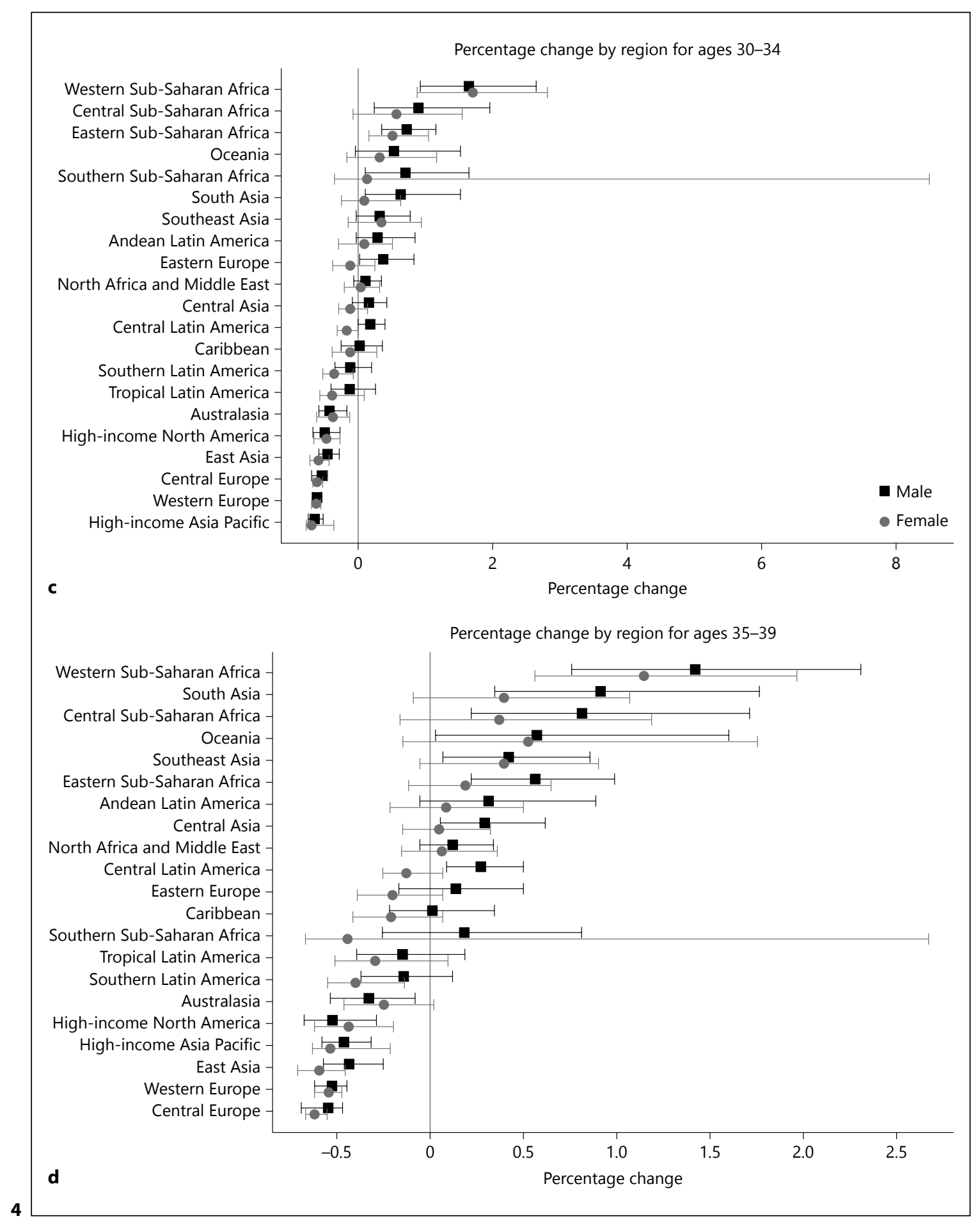

shown to be an independent contributor to stroke [23], with evidence suggesting it may be a more important mechanism in young adult stroke than in the older stroke population [24]. The risk and outcomes of stroke are also likely to vary across regions of the world due to differing environmental and genetic factors as well as varying levels of access to healthcare services [8]. Ethnic and racial differences may be important in early-onset stroke, with studies in the United States showing higher incidence of stroke and higher stroke mortality in those of Black 


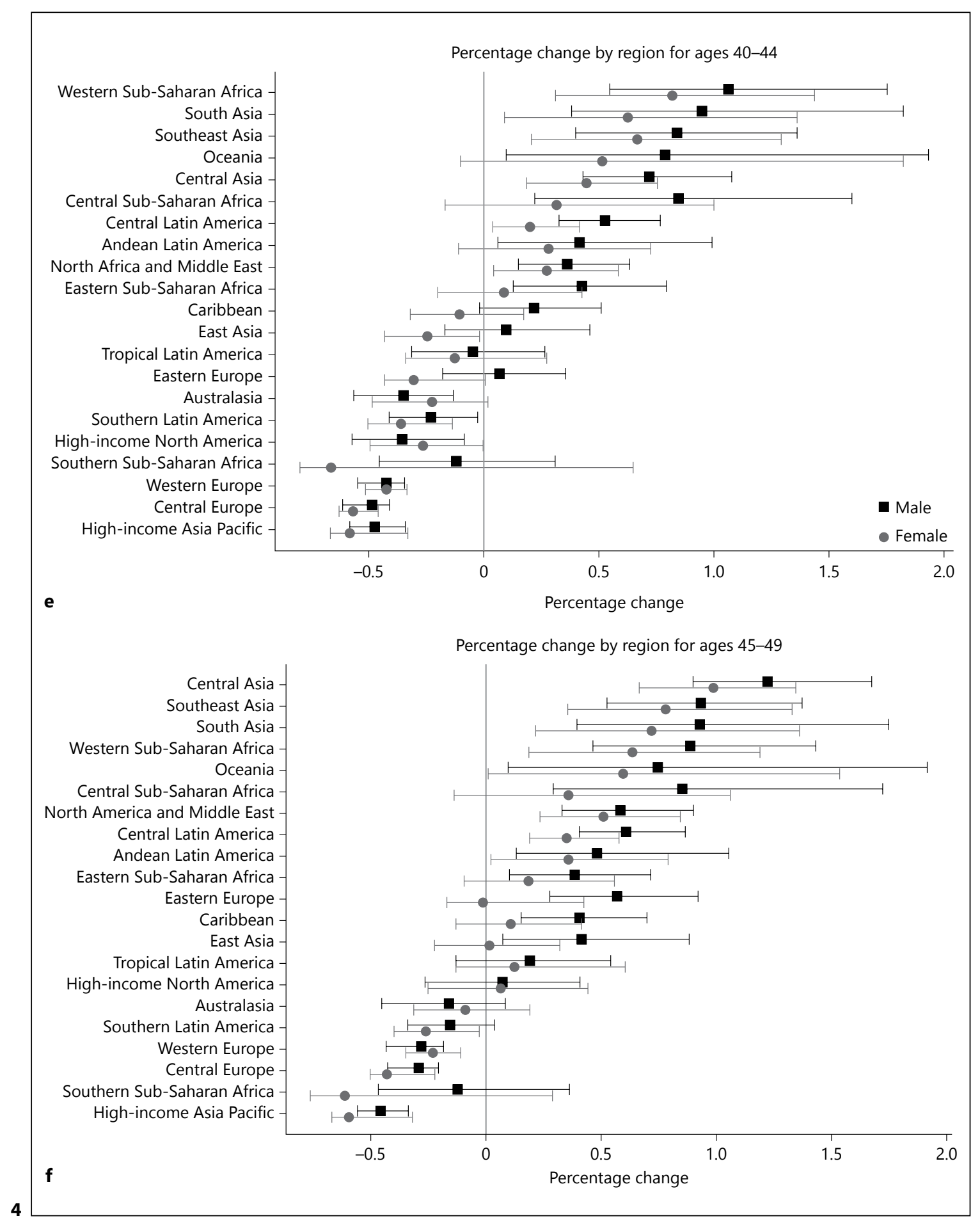

race compared to White race aged $20-45$ years [14]. The incidence of Moyamoya disease was shown to peak in a Chinese population at the age of 25-44 years [25].

However, recent evidence suggests that there is also an increasing association of stroke in younger adults with the prevalence of traditional vascular risk factors such as hypertension, diabetes and obesity [2]. The significant decline in death and DALY rates in developed countries may be the result of improved blood pressure control, acute care (e.g. stroke units, thrombolysis), increased 


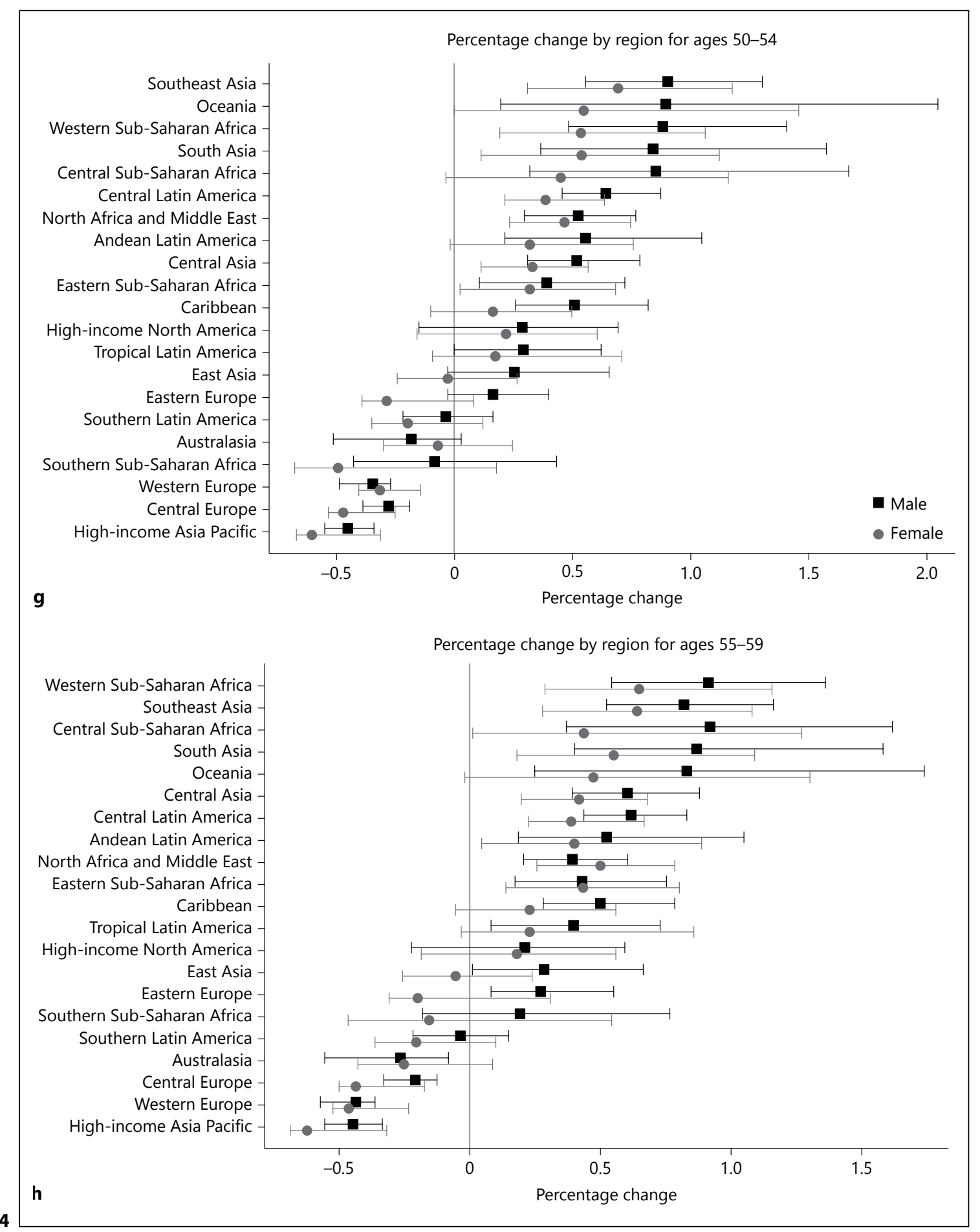

levels of neuroimaging leading to improved diagnosis and improvements in rehabilitation and chronic care [26-28]. For example, in a study of the safety and efficacy of thrombolysis in stroke patients of age 18-50 years, the prevalence of conventional stroke risk factors was high
$-27 \%$ had hypertension, $6 \%$ had diabetes and 21\% had hyperlipidemia [29]. In the multinational European Young Fabry Patients cohort of young adults aged 18-55 years, $55 \%$ were smokers, $48 \%$ were physically inactive, $46 \%$ had hypertension and $35 \%$ had dyslipidemia [30]. The 


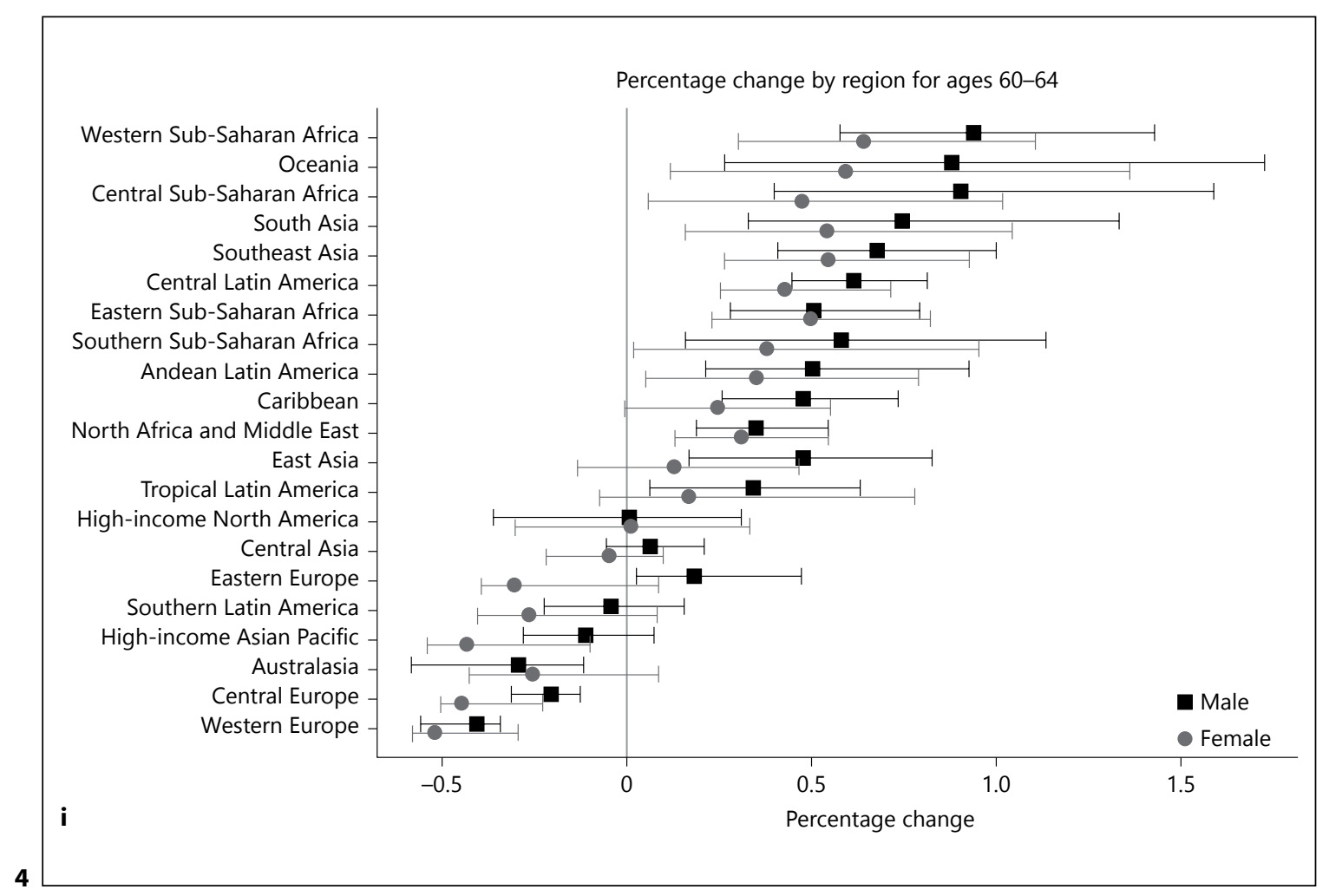

prevalence of risk factors has also been shown to differ between regions and ethnic groups. For example, hyperlipidemia and smoking appear to be the most common risk factor in young Chinese adults [31], while hypertension and diabetes are more prevalent in Black Americans [14]. The increased prevalence of modifiable risk factors for stroke may be a contributing factor to the increase in the proportion of younger adults experiencing stroke.

There are limitations to this analysis, which have been described previously $[10,11]$. Some country level data for young adult stroke was extrapolated from data from subnational regions, particularly in developing countries. Low-income countries are more likely to have missing data, due to lack of population-based studies, particularly in this younger age group. More data are needed on estimates of first-ever in a lifetime and recurrent incidence of stroke to provide a more complete picture of stroke burden and adequately gauge the success of prevention strategies in different regions of the world. While estimates of incidence were not available in the current study, these will be important outputs planned for the GBD 2015 Study. A major strength of this study was the use of consistent methods to enable comparison between subtypes of stroke, both with global and regional estimates. The production of estimates for all 5-year age categories al- lows us to report global estimates for the important category of younger adults with stroke.

The trend toward declining death and DALY rates developing countries is highly encouraging, but developing regions are still far behind compared to developed regions, in terms of gains in reducing overall stroke burden in the past 30 years. New strategies are needed to improve stroke surveillance efforts within lower income countries and support further efforts to estimate stroke burden for important subpopulations such as younger adults. These population-level estimates can encourage data-driven public health planning. Overall, improvements in acute and post-stroke care as well as continued investments in the reduction of traditional risk factors will be needed to reduce the burden of stroke in younger adults, particularly in developing countries.

\section{Acknowledgments}

This work was undertaken as a part of the GBD, Injuries, and Risk Factors (GBD 2013) study. The results in this paper are prepared independently of the final estimates of the GBD 2010 study. We thank Sarah Safranek, University of Washington Health Sciences Library, for her help in developing the systematic review literature search strategies; Hannah Gardener, University of Miami, 
for helping reviewing some studies; and Helen McDonald, AUT University National Institute for Stroke And Applied Neurosciences, for organizing teleconferences and secretarial support. The authors alone are responsible for the views expressed in this publication.

\section{Contributions}

R.K. wrote the first draft of the report. G.A.R. reviewed the first draft. V.L.F., G.A.R. and C.M. developed the study concept and oversaw the research. V.L.F., R.K. and G.A.R. undertook reviews of studies. M.H.F., M.N. and P.P. provided statistical analysis of the data and S.T. produced the graphs. M.H.F., M.N. and C.M. developed the statistical model and wrote a section on statistical analysis. All authors contributed to the critical revision of the manuscript for important intellectual content.

\section{Disclosure Statement}

All the authors declare that they have no conflicts of interest.

\section{Role of the Funding Source}

This study was funded by the Bill and Melinda Gates Foundation. The sponsor of the study had no role in the study design, data collection, data analysis, data interpretation or writing of the report. The Writing and GBD 2013 Global Analysis Group had access to all data sources and was responsible for the content of the report and the decision to submit for publication.

\section{Disclaimer}

The views expressed in this article are those of the authors and do not necessarily represent the views of the National Heart, Lung, and Blood Institute, National Institute of Mental Health, National Institutes of Health or the US Department of Health and Human Services.

\section{Appendix}

\section{GBD Stroke Panel Experts Group (in Alphabetical Order by} Country)

Argentina (Maria Cecilia Bahit); Australia (Amanda G. Thrift, Atte Meretoja, Bill Stavreski, Craig S. Anderson, Edwin Pearse, Geoffrey Donnan, Graeme J. Hankey, Mark T. MacKay, Stephen Davis, Zanfina Ademi; Austria (Michael Brainin); Azerbaijan (Tural Guliyev); Bahrain (Randah R. Hamadeh); Barbados (Heather Harewood, Karen Springer); Brazil (Iuri da Costa Leite, Jefferson Gomes Fernandes, Norberto Luiz Cabral, Paulo A. Lotufo); Bulgaria (Klara Dokova); Canada (Farshad Pourmalek, Gabrielle deVeber, Luciano A. Sposato, M. Patrice Lindsay, Patricia M. Riccio); Chile (Pablo M. Lavados); China (Bin Li, Chuanhua Yu, Guo-

hong Jiang, Jixiang Ma, Maigeng Zhou, Ming Liu, Shankuan Zhu, Wenzhi Wang, Xiaofeng Liang, Yong Zhang); Colombia (Gabriel Alcalá-Cerra); Denmark (Hanne K. Christensen, Thomas Truelsen); Egypt (Foad Abd-Allah); Ethiopia (Awoke Temesgen, Berhe Weldearegawi Sahle, Semaw Ferede Abera, Yohannes Adama Melaku); Fiji (Devina Nand); France (Maurice Giroud); Germany (Jost B. Jonas, Matthias Endres, Ronny Westerman); Greece (Konstantinos Stroumpoulis); India (Dorairaj Prabhakaran, Jeyaraj Durai Pandian, Man Mohan Mehndiratta, Nobhojit Roy, Panniyammakal Jeemon, Rajeev Gupta, Vasanthan Rajagopalan); Indonesia (Soewarta Kosen, Tati Suryati Warouw); Iran (Reza Malekzadeh); Ireland (Martin J. O’Donnell); Israel (David Tanne, Natan M. Bornstein); Italy (Stefano Ricci, Valeria Caso); Japan (Yoshihiro Kokubo, Yukito Shinohara); Jordan (Majed Masoud Asad); Kenya (Vitalis Kizito Bwire); Korea (Sun Ha Jee, Young-Ho Khang); Malaysia (Kim Yunjin, Ramesh Sahathevan); Mexico (Ismael Campos-Nonato); Morocco (Fortuné Gankpé); Myanmar (Chaw Yin Myint); Netherlands (Johanna M. Geleijnse); New Zealand (Priya Parmar, Rita V. Krishnamurthi, Suzanne Barker-Collo, Valery L. Feigin); Nigeria (Rufus Olusola Akinyemi); Norway (Ole Norheim); Qatar(ShamsEldin Khalifa); Russia (Michael Kravchenko, Michael Piradov, Nicolay Shalamov, Vasiliy Victorovich Vlassov, Yuri Varakin); Rwanda (Jean De Dieu Ngirabega, Jean Pierre Nyemazi, Marie Aimee Muhimpundu); Saudi Arabia (Mohammad Saeedi, Neeraj Bedi); Singapore (Narayanaswamy Venketasubramanian); South Africa (Andre Pascal Kengne); Spain (David Rojas-Rueda, Ferrán Catalá-López); Sri Lanka (Samath D. Dharmaratne); Sweden (Bo Norrving, Rasmus Havmoeller); Uganda (Leo Atwine); United Kingdom (Amitava Banerjee, Charles Wolfe, Derrick A. Bennett, Finbar O'Callaghan, Ivy Shiue, Julia A. Critchley, Majid Ezzati, Michael Soljak, Myles D. Connor, Peter M. Rothwell, Rajiv Chowdhury, Rustam Al-Shahi Salman, William Whiteley, Zhengming Chen); Uruguay (Mercedes Colomar); USA (Adnan M. Durrani, Anand Dayama, Andrew E. Moran, Awoke Misganaw, Brett M. Kissela, Catherine Amlie-Lefond, Catherine O. Johnson, Cheng Huang, Christopher Murray, Chugh Sumeet, Daniel Kim, David K. Cundiff, David Lawrence Tirschwell, Dhruv S. Kazi, Dima Qato, Edmond Kato Kabagambe, Eric Ding, Gene Bukhman, Gene Kwan, George A. Mensah, George D. Thurston, Grant Nguyen, Gregory A. Roth, Josef Coresh, Kate Lefondulq, Kevin N. Sheth, Matthew A. Corriere, Mohammad H. Forouzanfar, Mohsen Naghavi, Nana Mainoo, Norman J. Beauchamp, Ralph L. Sacco, Richard F. Gillum, Sanjay Basu, Stephen M. Schwartz, Sumeet Chugh, Teresa Fung, Theo Vos, Tim E. Byers, Uchechukwu K.A. Sampson, Walter A. Rocca, Warren Lo).

References

Neuroepidemiology 2015;45:190-202 DOI: $10.1159 / 000441098$
Stroke Prevalence, Mortality and DALYs

in Adults Aged 20-64 Years in 1990-2013
1 Kissela BM, Khoury JC, Alwell K, Moomaw CJ, Woo D, Adeoye O, Flaherty ML, Khatri P, Ferioli S, De Los Rios La Rosa F, Broderick JP Kleindorfer DO: Age at stroke: temporal trends in stroke incidence in a large, biracial population. Neurology 2012;79:1781-1787.

$\checkmark 2$ Kittner SJ, Singhal AB: Premature atherosclerosis: a major contributor to early-onset ischemic stroke. Neurology 2013;80:1272-1273.

-3 Wang Y, Rudd AG, Wolfe CD: Age and ethnic disparities in incidence of stroke over time: the South London stroke register. Stroke 2013;44:3298-3304. 
-4 Metso AJ, Metso TM, Putaala J, Tatlisumak T: Stroke in young adults: every saga has a beginning. Eur J Neurol 2010;17:1317.

5 Feigin VL, Forouzanfar MH, Krishnamurthi $\mathrm{R}$, Mensah GA, Connor M, Bennett DA, Moran AE, Sacco RL, Anderson L, Truelsen T, O’Donnell M, Venketasubramanian N, Barker-Collo S, Lawes CM, Wang W, Shinohara Y, Witt E, Ezzati M, Naghavi M: Global and regional burden of stroke during 1990-2010: findings from the global burden of disease study 2010. Lancet 2014;383:245254.

6 Marini C, Russo T, Felzani G: Incidence of stroke in young adults: a review. Stroke Res Treat 2010;2011:535672.

7 Goeggel Simonetti B, Mono ML, Huynh-Do U, Michel P, Odier C, Sztajzel R, Lyrer P, Engelter ST, Bonati L, Gensicke H, Traenka C, Tettenborn B, Weder B, Fischer U, Galimanis A, Jung S, Luedi R, de Marchis GM, Weck A, Cereda CW, Baumgartner R, Bassetti CL, Mattle HP, Nedeltchev K, Arnold M: Risk factors, aetiology and outcome of ischaemic stroke in young adults: the Swiss young stroke study (SYSS). J Neurol 2015;262:20252032.

-8 Maaijwee NA, Rutten-Jacobs LC, Schaapsmeerders P, van Dijk EJ, de Leeuw FE: Ischaemic stroke in young adults: risk factors and long-term consequences. Nat Rev Neurol 2014; 10:315-325.

$\checkmark 9$ de Los Ríos F, Kleindorfer DO, Khoury J, Broderick JP, Moomaw CJ, Adeoye O, Flaherty ML, Khatri P, Woo D, Alwell K, Eilerman J, Ferioli S, Kissela BM: Trends in substance abuse preceding stroke among young adults: a population-based study. Stroke 2012; 43:3179-3183.

10 Feigin VL, Krishnamurthi RV, Parmar P, Norrving B, Mensah GA, Bennett DA, Barker-Collo S, Moran AE, Sacco RL, Truelsen T, Davis S, Pandian JD, Naghavi M, Forouzanfar MH, Nguyen G, Johnson C, Vos T, Meretoja A, Murray CJL, Roth GA: Update on the global burden of ischemic and hemorrhagic stroke in 1990-2013: the GBD 2013 study. Neuroepidemiology 2015;45:161-176.

11 Roth GA, Johnson CO, Nguyen G, Naghavi M, Feigin VL, Murray CJL, Forouzanfar MH, Vos T: Methods for estimating the global burden of cerebrovascular diseases. Neuroepidemiology 2015;45:146-151.
12 Naghavi M, Wang H, Lozano R, Davis A, et al: Global, regional, and national age-sex specific all-cause and cause-specific mortality for 240 causes of death, 1990-2013: a systematic analysis for the global burden of disease study 2013. Lancet 2015;385:117-171.

13 Flaxman AD, Vos T, Murray CJL (eds): An Integrative Metaregression Framework for Descriptive Epidemiology. University of Washington Press, 2015.

14 Chong JY, Sacco RL: Epidemiology of stroke in young adults: race/ethnic differences. J Thromb Thrombolysis 2005;20:77-83.

15 Béjot Y, Daubail B, Jacquin A, Durier J, Osseby GV, Rouaud O, Giroud M: Trends in the incidence of ischaemic stroke in young adults between 1985 and 2011: the Dijon stroke registry. J Neurol Neurosurg Psychiatry 2014;85: 509-513.

16 Sultan S, Elkind MS: The growing problem of stroke among young adults. Curr Cardiol Rep 2013;15:421.

17 de-Graft Aikins A, Boynton P, Atanga LL: Developing effective chronic disease interventions in Africa: insights from Ghana and Cameroon. Global Health 2010;6:6.

18 Nilanont Y, Nidhinandana S, Suwanwela NC, Hanchaiphiboolkul S, Pimpak T, Tatsanavivat P, Saposnik G, Poungvarin N: Quality of acute ischemic stroke care in Thailand: a prospective multicenter countrywide cohort study. J Stroke Cerebrovasc Dis 2014;23:213-219.

9 Bushnell C: Introduction: stroke in young adults. J Thromb Thrombolysis 2005;20:75.

20 Spengos K, Vemmos K: Risk factors, etiology, and outcome of first-ever ischemic stroke in young adults aged 15 to 45 - the Athens young stroke registry. Eur J Neurol 2010;17:13581364.

21 Adams HP Jr, Kappelle LJ, Biller J, Gordon DL, Love BB, Gomez F, Heffner M: Ischemic stroke in young adults. Experience in 329 patients enrolled in the Iowa registry of stroke in young adults. Arch Neurol 1995;52:491-495.

22 Ji R, Schwamm LH, Pervez MA, Singhal AB: Ischemic stroke and transient ischemic attack in young adults: risk factors, diagnostic yield, neuroimaging, and thrombolysis. JAMA Neurol 2013;70:51-57.

23 Rolfs A, Fazekas F, Grittner U, Dichgans M, Martus P, Holzhausen M, Böttcher T, Heuschmann PU, Tatlisumak T, Tanislav C, Jungehulsing GJ, Giese AK, Putaala J, Huber R, Bo- dechtel U, Lichy C, Enzinger C, Schmidt R, Hennerici MG, Kaps M, Kessler C, Lackner K, Paschke E, Meyer W, Mascher H, Riess O, Kolodny E, Norrving B; Stroke in Young Fabry Patients (sifap) Investigators: Acute cerebrovascular disease in the young: the stroke in young fabry patients study. Stroke 2013;44: 340-349.

24 Brey RL: Antiphospholipid antibodies in young adults with stroke. J Thromb Thrombolysis 2005;20:105-112.

25 Duan L, Bao XY, Yang WZ, Shi WC, Li DS, Zhang ZS, Zong R, Han C, Zhao F, Feng J: Moyamoya disease in China: its clinical features and outcomes. Stroke 2012;43:56-60.

26 Grau AJ, Eicke M, Biegler MK, Faldum A, Bamberg C, Haass A, Hardt R, Hufschmidt A, Lowitzsch K, Marx J, Schmitt E, Schoenemann H, von Arnim W, Weiss H, Dienlin S: Quality monitoring of acute stroke care in Rhineland-Palatinate, Germany, 2001-2006. Stroke 2010;41:1495-1500.

27 Lakshminarayan K, Berger AK, Fuller CC, Jacobs DR Jr, Anderson DC, Steffen LM, Sillah A, Luepker RV: Trends in 10-year survival of patients with stroke hospitalized between 1980 and 2000: the Minnesota stroke survey. Stroke 2014;45:2575-2581.

28 Putaala J, Metso AJ, Metso TM, Konkola N, Kraemer Y, Haapaniemi E, Kaste M, Tatlisumak T: Analysis of 1008 consecutive patients aged 15 to 49 with first-ever ischemic stroke: the Helsinki young stroke registry. Stroke 2009;40:1195-1203.

29 Toni D, Ahmed N, Anzini A, Lorenzano S, Brozman M, Kaste M, Mikulik R, Putaala J, Wahlgren $\mathrm{N}$ : Intravenous thrombolysis in young stroke patients: results from the SITSISTR. Neurology 2012;78:880-887.

30 Von Sarnowski B, Putaala J, Grittner U, Gaertner B, Schminke U, Curtze S, Huber R, Tanislav C, Lichy C, Demarin V, Basic-Kes V, Ringelstein EB, Neumann-Haefelin T, Enzinger C, Fazekas F, Rothwell PM, Dichgans M, Jungehulsing GJ, Heuschmann PU, Kaps M, Norrving B, Rolfs A, Kessler C, Tatlisumak $\mathrm{T}$ : Lifestyle risk factors for ischemic stroke and transient ischemic attack in young adults in the stroke in young fabry patients study. Stroke 2013;44:119-125.

31 Lee TH, Hsu WC, Chen CJ, Chen ST: Etiologic study of young ischemic stroke in Taiwan. Stroke 2002;33:1950-1955. 


\section{Erratum}

In the article by Krishnamurthi RV, Moran AE, Feigin VL, Barker-Collo S, Norrving B, Mensah GA, Taylor S, Naghavi M, Forouzanfar MH, Nguyen G, Johnson CO, Vos T, Murray CJ, Roth GA, and the GBD 2013 Stroke Panel Experts Group, entitled 'Stroke prevalence, mortality and disability-adjusted life years in adults aged 20-64 years in 1990-2013: data from the Global Burden of Disease 2013 Study' [Neuroepidemiology 2015;45:190202, DOI: $10.1159 / 000441098$ ], the value $92,665,770$ needs to be corrected to $2,665,770$ in the paragraph on trends 1990-2013. The corrected paragraph is as follows:

Trends 1990-2013. Globally, there were significant increases in both numbers of cases (table 1) and prevalence rates of both HS and IS between 1990 and 2013 (fig. 1). The number of prevalent HS cases increased significantly in developing countries between $1990(1,450,240$ (95\% UI 1,391,277-1,510,464)) and 2013 (2,665,770 (95\% UI 2,521,485$2,789,120))$. 